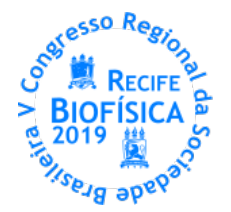

\title{
RESPOSTAS CARDIORRESPIRATÓRIAS DE CRIANÇAS SAUDÁVEIS SUBMETIDAS AO TESTE DE DEGRAU DE 3 MINUTOS
}

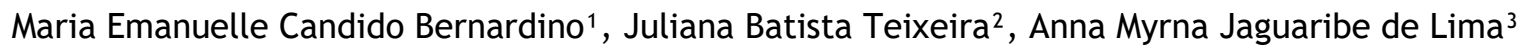 \\ Departamento de Biologia, UFRPE ${ }^{1}$; Departamento de Fisioterapia, UFPE²; Departamento de Morfologia Fisiologia Animal, UFRPE ${ }^{3}$ \\ *maria.emanuelle@hotmail.com
}

\begin{abstract}
INTRODUÇÃO
Um estilo de vida que envolve a prática regular de exercício físico, fornece uma melhor qualidade de vida e favorece a redução de doenças futuras. Em decorrência, no ponto de vista da saúde preventiva, propiciar o exercício físico à criança e ao adolescente promove uma redução de sedentarismo na vida adulta (LAZZOLI et al. 1998). Assim, avaliar a capacidade funcional de exercício é uma importante ferramenta clínica na avaliação dos sistemas metabólico e cardiorrespiratório, obtendo respostas cardiorrespiratórias ao esforço físico. Para este fim, são utilizados vários testes e uma forma de avaliar a tolerância ao exercício é de maneira direta, considerada o método padrão-ouro o teste cardiopulmonar de exercício, qual, é mensurado o consumo máximo de oxigênio $\left(\mathrm{VO}_{2 \text { máx }}\right)$. Este teste é considerado difícil de ser executado, por ter intensidade máxima, além de utilizar equipamentos caros e necessitar do recrutamento de pessoal especializado. A outra forma de avaliar a capacidade funcional de exercício é a de medida indireta, através de testes de campo ou de laboratório, sendo mais práticos de serem realizados e melhor tolerados pela população. Neste contexto, o teste de degrau é de fácil aplicação, baixo custo e considerado portátil, e consiste em subir e descer o degrau por um tempo determinado. Do ponto de vista fisiológico, o TD acarreta uma maior demanda metabólica e ventilatória em comparação com o teste de caminhada (SWINBURN et al.1989; Dal CORSO et al.2007; MARRARA et al.2012). Sendo assim, o teste é importante por fornecer diversas variáveis de informações sobre as respostas do sistema cardiovascular, atuando como indicativo de doenças do sistema cardiorrespiratório sendo indispensável nas práticas clínicas tanto em crianças doentes quanto saudáveis. Os objetivos desse trabalho foi avaliar as respostas cardiorrespiratórias de crianças saudáveis submetidas ao teste de degrau de três minutos (TD3M).
\end{abstract}

\section{MATERIAIS E MÉTODOS}

Foram avaliados 36 voluntários, que preencheram uma ficha de avaliação contendo as informações pessoais, dados antropométricos e a medida dos sinais vitais em repouso: pressão arterial (PA), saturação de oxigênio $\left(\mathrm{SpO}_{2}\right)$, frequências cardíaca $(\mathrm{FC})$ e respiratória (FR). Também foi preenchido pelos pais e pelas crianças o questionário Internacional de Atividade Física (IPAQ) Forma Curta, relativo ao nível de atividade física, Estas têm como objetivo a coleta de informações acerca da quantidade de dias semanais e de minutos ou horas gastas em atividades de esforço físico vigoroso e moderadas, e também caminhada e ficar sentado. E após o preenchimento é possível classificar a criança nas modalidades: sedentário, irregularmente ativo, ativo e muito ativo. A realização dos testes está sendo feita em dois dias, com no mínimo dois e no máximo sete dias de intervalo entre eles, o TD3M é realizado através de um degrau de madeira. Os voluntários são orientados a subir e descer 24 vezes por minuto (96 batidas por minuto) mantendo-se num ritmo orientando por um metrônomo, durante o tempo de três minutos pertinentes ao teste Os parâmetros mensurados foram: frequência cardíaca $(F C)$, pressão arterial sistólica (PAS), pressão arterial diastólica (PAD), fadiga de membros inferiores (avaliada pela Escala de Borg), e saturação de oxigênio $\left(\mathrm{SpO}_{2}\right)$ em repouso, durante, imediatamente após os testes e na fase de recuperação. [Os testes são interrompidos caso: o voluntário atinja a frequência cardíaca máxima estimada], ou seja, apresentados sinais de fadiga excessiva, ou a pedido do próprio voluntário. Caso isso aconteça, o voluntário será orientado a parar e descansar em posição ortostática, porém o cronômetro continuará ligado, podendo retomar o teste assim que possível.

\section{RESULTADOS}

Foram analisadas 36 crianças na faixa etária de 7-11 anos. As características gerais da amostra foram descritas na tabela 1. 63,8\% da amostra é composta de meninas. Sobre o estado nutricional, se tem uma distribuição proporcional de indivíduos considerados como normais, sobrepeso e obesidade. Quanto ao nível de atividade física das crianças, o IPAQ, respondido pelas crianças pelos pais a maior parte da amostra foi classificada como inativa.

Tabela1. Características gerais da amostra

Dados expressos como média e desvio padrão e percentagem.

IMC: índice de massa corporal. Comprimento de MMI: comprimento de membros inferiores.

\begin{tabular}{lc}
\hline & Total $(\mathrm{n}=36)$ \\
Sexo & \\
Menina & $23(63,8 \%)$ \\
Menino & $13(36,11 \%)$ \\
Idade, anos & $9,58 \pm 1,180$ \\
Peso, $\mathrm{kg}$ & $39,22 \pm 10,889$ \\
Altura, $\mathrm{m}$ & $1,31 \pm 0,467$ \\
IMC, $\mathrm{kg} / \mathrm{m} 2$ & $18,86 \pm 3,563$ \\
Comprimento de MMII & $83,17 \pm 7,489$ \\
Estado Nutricional, $\mathrm{n}$ (\%) & $1,67 \pm 0,793$ \\
Normal & $18,5-24,9 \%$ \\
Sobrepeso & $25,0-29,9 \%$ \\
Obesidade & $30,0-34,9 \%$ \\
Nível de Atividade física - Pais $\mathrm{n}(\%)$ & Total $(\mathrm{n}=16)$ \\
Inativo & $10(62,5 \%)$ \\
Ativo & $6(37,5 \%)$ \\
Nível de Atividade física - Criança $\mathrm{n}(\%)$ & Total $(\mathrm{n}=36)$ \\
Inativo & $24(66,7 \%)$ \\
Ativo & $12(33,3 \%)$ \\
\hline
\end{tabular}


Na tabela 2, são os valores da frequência cardíaca em Repouso e a frequência cardíaca máxima obtida em crianças submetidas ao TD3M.

Tabela 2:Valores de $\mathrm{FC}_{\text {Repouso; }}$ e $F C_{\text {Máx obtida }}$ em crianças submetidas ao TD3M FCRepouso: frequência cardíaca de repouso; FC Máx. Obtida: frequência cardíaca máxima obtida

\begin{tabular}{cc}
\hline & (bpm) \\
\hline $\mathrm{FC}_{\text {Repouso }}$ & $93,28 \pm 11,985$ \\
$\mathrm{FC}$ Máx. obtida & $141,00 \pm 15,045$ \\
\hline
\end{tabular}

$\mathrm{Na}$ tabela 3, é possível observar que não houve diferença significativa nos valores da frequência cardíaca e de fadiga dos membros inferiores. A saturação de oxigênio foi mais baixa no $2^{\circ}$ minuto de realização do teste.

Tabela 3. Comparação dos valores de FC, SpO2 e fadiga de membros inferiores antes, durante e após a realização do TD3M

FC (bpm):frequência cardíaca. $\mathrm{SPO}_{2} \%$ : percentual da saturação periférica de oxigênio; Fadiga de MMIl: fadiga de membros inferiores.

\begin{tabular}{lllll} 
& Repouso & $\begin{array}{l}\text { Durante }\left(2^{\circ}\right. \\
\text { Minuto) }\end{array}$ & 1'Recup & Valor de $\mathrm{p}$ \\
$\mathrm{FC}(\mathrm{bpm})$ & $93,2 \pm 11,9$ & $133,3 \pm 25,4$ & $96,0 \pm$ & $\mathrm{P}=0,12$ \\
$\mathrm{SpO}_{2}(\%)$ & $98,2 \pm 0,8$ & $96,0 \% \pm 4,1^{*}$ & 16,9 & $98,4 \% \pm 0,7$ \\
$\begin{array}{l}\text { Fadiga de } \\
\text { MMII }\end{array}$ & $0,83 \pm 1,82$ & $3,44 \pm 3,18$ & $1,92 \pm 2,33$ & $\mathrm{p}=0,01$ \\
\hline
\end{tabular}

$\mathrm{Na}$ Tabela 4, é observado que a pressão arterial sistólica foi mais elevada no primeiro minuto de recuperação e a pressão arterial diastólica volta aos níveis de repouso no primeiro minuto de recuperação.

Tabela 4. Comparação dos valores de PAS e PAD antes e após a realização do TD3M. PAS: Pressão Arterial Sistólica. PAD: Pressão arterial Diastólica

\begin{tabular}{lccc}
\hline & Repouso & 1'Recup & Valor de $\mathrm{p}$ \\
\hline PAS $(\mathrm{mmHg})$ & $95,83 \pm 9,063$ & $100,08 \pm 13,2$ & $\mathrm{p}=0,02$ \\
PAD $(\mathrm{mmHg})$ & $63,72 \pm 6,935$ & $64,14 \pm 6,095$ & $\mathrm{p}=0,00$ \\
\hline
\end{tabular}

\section{DISCUSSÃO}

Neste estudo, foram avaliadas as respostas cardiorrespiratórias de crianças saudáveis em um teste de degrau de três minutos (TD3M). Os resultados encontrados no nosso estudo mostram que não houve uma diferença significativa na FC e na Fadiga de MMII. Na saturação de oxigênio, os níveis baixaram no segundo minuto durante o teste. De acordo com Basso et al. (2010), referente à comparação do desempenho físico e das respostas obtidas nos testes de caminhada de seis minutos (TC6) e do degrau de seis minutos (TD6) entre adolescentes asmáticos e saudáveis, observaram-se valores significativamente maiores na pressão sistólica (PAS) no TD6. Com o término do esforço físico e a redução da demanda metabólica, os níveis de PAS e PAD tendem a voltar pros níveos de normalidade nos indivíduos saudáveis (PASSARO 1997).

\section{CONCLUSÃO}

Podemos concluir que, o TD3M excede a demanda regulatória do organismo causando um estresse cardiovascular e possibilitando a avaliação da tolerância ao esforço em crianças saudáveis.

\section{REFERÊNCIAS}

BASSO, Renata; JAMAMI, mauricio et al. Avaliação da capacidade de exercício em adolescentes asmáticos e saudáveis. Disponível em < http://www.scielo.br/pdf/rbfis/v14n3/05.pdf>

LAZZOLI, José; NÓBREGA, Antonio et al. Atividade física e saúde na infância e adolescência. Disponível em < http: / / www.scielo.br/scielo.php?script=sci_arttext\&pid=S1517-

86921998000400002>
PASSARO , L; Resposta cardiovascular na prova de esforço: pressão arterial sistólica. Disponível em http://www.scielo.br/scielo.php?script=sci_arttext\&pid=S151786921997000100003>

SWINBURN et al.1989; Dal CORSO et al.2007; MARRARA et al.2012 Responsividade do teste do degrau de seis minutos a um programa de treinamento físico em pacientes com DPOC. Disponível em: <http: / / www.scielo.br/scielo.php?script=sci_\&tid=S180637132012000500007> 